\title{
Thermal reflow engineered cylindrical polymer waveguides for optical interconnects
}

\author{
Aleksandrs Marinins, Oskars Ozolins, Xiaodan Pang, Aleksejs Udalcovs, Jaime Rodrigo Navarro, \\ Aditya Kakkar, Richard Schatz, Gunnar Jacobsen and Sergei Popov
}

\begin{abstract}
Integrated polymer photonics brings low cost and high fabrication flexibility to optoelectronic industry. However, this platform needs to overcome several issues to be efficient enough for practical applications. In this work, we experimentally demonstrate a decrease of propagation losses and polarization sensitivity of polymer waveguide-based devices as a result of thermal treatment. Heating of poly(methyl methacrylate) (PMMA) strip optical waveguides above the glass transition temperature initiates a waveguide surface reflow due to a decrease of a polymer viscosity and surface tension energy. This results in a decrease of surface roughness and shape change from rectangular to cylindrical; thus, scattering losses and polarization sensitivity are minimized.
\end{abstract}

Index terms - Integrated optics; Optical polymers; Optical interconnections.

\section{INTRODUCTION}

$\mathrm{O}$ PTICAL polymers have attracted research and industry attention for many decades. Nowadays, optical polymer based photonic integrated circuits (PICs) are highly demanded by optoelectronic applications [13]. They offer transparency in visible and near-IR, wide tunability for particular applications, low-cost fabrication and simple packaging [4]. Major requirements for on-chip optical signal transmission and processing are low propagation losses and polarization dependent losses (PDL) [2, 5]. Several groups successfully developed polymer buried channel waveguides [6-8] and chalcogenide glass rib waveguides [9] which fulfill aforementioned requirements. However, these platforms suffer from low core-cladding refractive index contrast and specific rib waveguide geometry, respectively. Both features lead to a limited integration density due to crosstalks, large footprint and increased bending radii; this also complicates a PIC design.

In this letter, we demonstrate a method to overcome low-contrast and geometry-related issues to develop low-

This work was funded by EU project ICONE (grant no 608099), EU project GRIFFON (grant no. 324391), Swedish ICT-TNG program, and Vetenskapsrädet project PHASE (grant no. 2016-04510). The equipment was funded by the Knut and Alice Wallenberg foundation.

A. Marinins, Aditya Kakkar, R. Schatz and S. Popov are with the School of Engineering Sciences, KTH Royal Institute of Technology, SE 10044, Stockholm, Sweden (e-mail: marinin@kth.se; adityak@kth.se; rschatz@kth.se, sergeip@kth.se).

O. Ozolins, X. Pang, A. Udalcovs, J. Navarro and G. Jacobsen are with the Networking and Transmission Laboratory (NETLAB), RISE Acreo AB, SE 16425, Kista, Sweden (e-mail: oskars.ozolins@ri.se; xiaodan.pang@ri.se; aleksejs.udalcovs@ri.se; jaime.rodrigonavarro@ri.se; gunnar.jacobsen@ri.se). loss polarization insensitive compact waveguides. We fabricated single-mode polymer waveguides and microring resonators with mostly air $(\mathrm{n}=1)$ as a cladding material to maximize an index contrast and thermally reflowed them to decrease surface roughness induced scattering losses [10]. Moreover, heating changes a waveguide polymer viscosity, surface tension energy turns waveguide shape from rectangular to cylindrical [11, 12], reducing polarization sensitivity. Propagation losses and PDL of prepared waveguides were assessed; filtering properties of microring resonators analyzed. Optical interconnect performance of demonstrated waveguides was evaluated by transmitting high speed 28 Gbaud 64-QAM signal. PMMA was chosen because of its availability, high transparency at telecom wavelengths and low glass transition temperature $\left(\mathrm{T}_{\mathrm{g}}\right)$ [13]. Relative approach was implemented on $\mathrm{SiO}_{2}$ waveguides; however, fabrication process is difficult due to high $\mathrm{SiO}_{2}$ melting point $[14,15]$.

\section{DESIGN AND FABRICATION}

Finite element method 2D simulation in COMSOL Multiphysics environment was performed to estimate waveguide geometry supporting single-mode behavior. Waveguide diameter of $2.5 \mu \mathrm{m}$ shows a single-mode light propagation and a good field confinement (Fig. 1a).
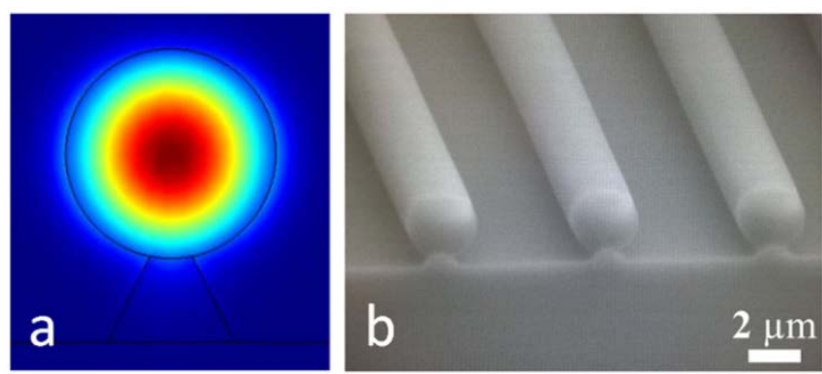

Fig. 1. Waveguide mode field distribution simulation (a) and scanning electron microscopy (SEM) image of prepared waveguides (b).

As it can be seen, fabricated waveguides have almost perfectly circular core profile making simulated field distribution very close to reality (Fig. 1b).

To realize the proposed waveguides, $2.5 \mu \mathrm{m}$ thick PMMA (Microchem Corp.) core film was spin coated on a Si wafer with $10 \mu \mathrm{m}$ thermally grown oxide layer (Fig. 2). The wafer was cleaned in acetone and isopropanol and subsequently dehydrated on a hotplate at $180^{\circ} \mathrm{C}$ for 10 minutes. Then a $1.8 \mu \mathrm{m}$ thick imaging photoresist Shipley S1818 was spin coated and exposed with h-line $(405 \mathrm{~nm}$, $100 \mathrm{~mJ} / \mathrm{cm}^{2}$ ) radiation on a Karl Suss MJB3 mask aligner. 
PMMA and imaging resist spin coating

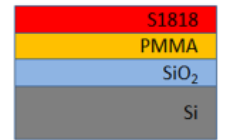

$\downarrow$

H-line lithography \& development

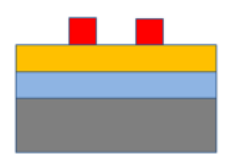

$\downarrow$

$$
\mathrm{O}_{2} \mathrm{RIE}
$$

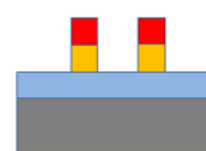

Fig. 2. Waveguide fabrication steps.

After development in MF-319 (Microchem Corp.) waveguide pattern was transferred to the PMMA core layer with $\mathrm{O}_{2}$ reactive ion etching (RIE). Plasma etching (Oxford Plasmalab 80) was processed with 80W RF power and 5 mTorr pressure to obtain high etch anisotropy. The PMMA etch rate was $\sim 200 \mathrm{~nm} / \mathrm{min}$ with a photoresist selectivity of $\sim 3: 1$, photoresist remains were floodexposed to $405 \mathrm{~nm}$ light and removed with MF-319. Next, $\mathrm{SiO}_{2}$ cladding was isotropically etched in buffered hydrofluoric acid (BHF) forming waveguides on pedestals; this step enhanced waveguide light confinement [16, 17]. Finally, waveguides were heated on a hotplate to reflow surface and to form a cylindrical shape. The optimized thermal treatment conditions are $135^{\circ} \mathrm{C}$ for $30 \mathrm{~s}$.

Waveguide reshaping process also brings the effect of feature size change: waveguide width changes upon transfer from rectangular to circular shape. The width expansion is easily calculated from cross-sectional geometry considering constant area:

$$
w h=\pi\left(\frac{d}{2}\right)^{2}(1)
$$

where $w$ and $h$ are rectangle width and height, respectively, and $d$ is a circle diameter (Fig. 3a). If we input $2 \mu \mathrm{m}$ for $w$ and $2.5 \mu \mathrm{m}$ for $h$, the $d$ is $\sim 2.53 \mu \mathrm{m}$, $\sim 530 \mathrm{~nm}$ wider than initial structure. This allows using contact lithography with resolution beyond its limit. Particularly in this work a gap between the bus waveguide and the ring resonator was narrowed from $900 \mathrm{~nm}$ to $\sim 370$ $\mathrm{nm}$ which dramatically improved coupling to a resonator (Fig. 3b). By making higher aspect ratio waveguides it is possible to achieve even narrower gap.

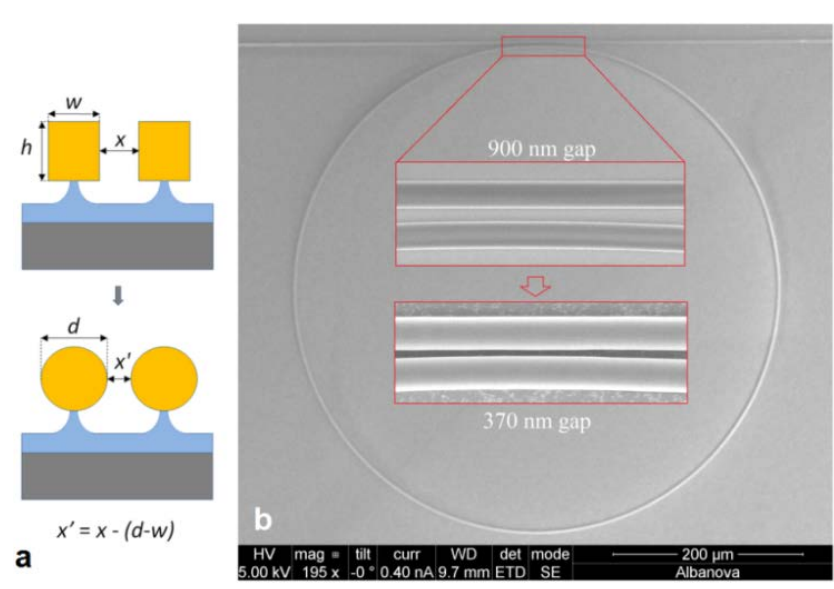

Fig. 3. Gap narrowing scheme (a) and SEM image of a microring resonator (b). Insets show magnified coupling region before (top) and after reflow (bottom).

\section{CHARACTERIZATION}

We fabricated and evaluated cylindrical waveguides, ring resonators based on proposed waveguides and a spiral $10 \mathrm{~cm}$ long waveguide to check their performance at high speed transmission link (using a 28 Gbaud Nyquist-shaped optical 64-QAM signal).

Cylindrical waveguides were compared to non-reflown rectangular ones. Standard lensed fiber coupling setup with tunable C-band laser (Agilent 8164A), polarization controller and optical spectrum analyzer (Agilent 86082A) was employed for device analysis. Cut-back propagation loss measurements were performed with 10, 7 and $3 \mathrm{~mm}$ long waveguides on cleaved chips. TE and TM modes were launched to estimate PDL. Table 1 depicts propagation losses at $\lambda=1550 \mathrm{~nm}$.

Table 1. Measured propagation losses

\begin{tabular}{lccc}
\hline & $\begin{array}{c}\text { TE, } \\
\text { dB/cm }\end{array}$ & TM, dB/cm & $\begin{array}{c}\text { PDL } \\
\text { |TE-TM|, } \\
\text { dB/cm }\end{array}$ \\
\hline $\begin{array}{l}\text { Cylindrical } \\
\text { waveguides }\end{array}$ & $1.32 \pm 0.21$ & $1.40 \pm 0.20$ & $\sim 0.08$ \\
$\begin{array}{l}\text { Rectangular } \\
\text { waveguides }\end{array}$ & $1.95 \pm 0.21$ & $1.66 \pm 0.19$ & $\sim 0.29$ \\
\hline
\end{tabular}

Each waveguide showed nearly negligible propagation loss variation from 1535 to $1575 \mathrm{~nm}$. Measurement error arises from fiber-chip coupling efficiency variations, material impurities and fabrication imperfections. We report significant improvement of both propagation losses and PDL of reflown waveguides. Thermal treatment nearly excludes a contribution of surface roughness induced losses leaving intrinsic material absorption and imperfection generated losses. PDL is reduced to less than $0.1 \mathrm{~dB} / \mathrm{cm}$ across the whole C-band, this makes demonstrated waveguides proper building blocks for polarization insensitive ring resonators, interferometers, directional couplers and high speed interconnects. Nevertheless, propagation losses are still too high for real applications mostly due to specific polymer molecule absorption [2]. We demonstrate waveguide performance enhancement as a result of the thermal treatment, this approach could be used with advanced low-loss optical 
polymers bringing optical devices of remarkable efficiency [18]. Microring resonators were fabricated with discussed cylindrical waveguide technology.
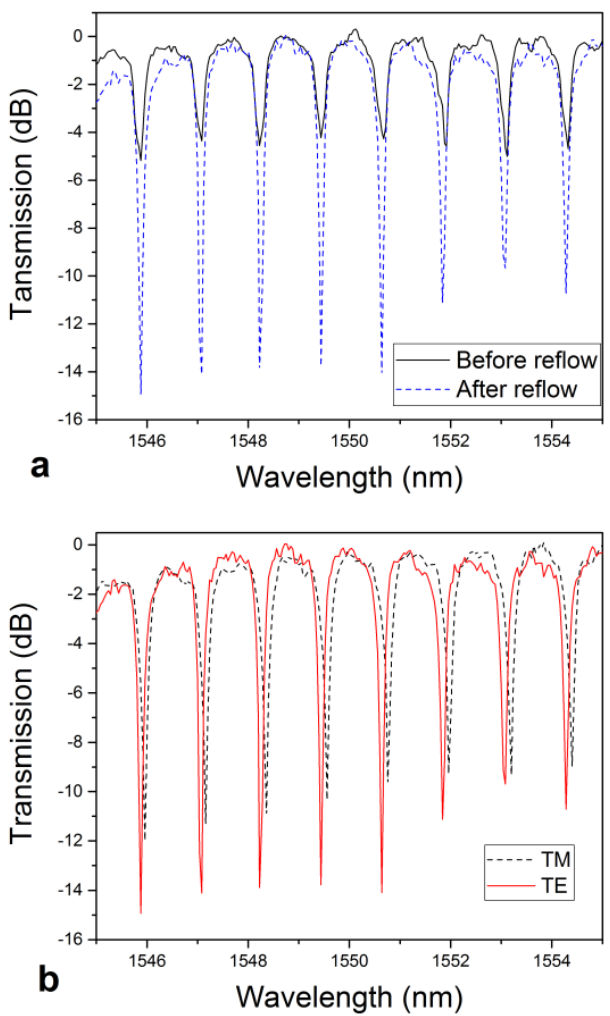

Fig. 4. Normalized transmission of a $250 \mu \mathrm{m}$ radius microring before and after reflow for TE mode (top), TE and TM mode transmission of reflown resonator (bottom).

Several groups have reported a successful fabrication of polymer microring and microracetrack resonators by electron beam lithography [19, 20] and nanoimprint lithography [16, 21], however, contact optical lithography is still the most cost-effective technique for polymer PICs [22]. Analogically to straight waveguides, microring resonators composed of rectangular and cylindrical waveguides were compared. It is seen that reflow induced coupling gap narrowing increased a coupling efficiency between a bus waveguide and a microring (Fig. 4a). A notch depth changed from $\sim-5 \mathrm{~dB}$ to $\sim-14 \mathrm{~dB}$ leading closer to a critical coupling condition. There is a significant improvement in terms of quality factor: it increased from $6.41 \times 10^{3}$ to $1.55 \times 10^{4}$ indicating notable cavity loss decrease.

Considering low PDL of cylindrical waveguides, polarization sensitivity of ring resonators was evaluated. As it is seen from Fig. 4b, TE and TM polarizations show different resonance positions and depths. The peak shift is $0.11 \mathrm{~nm}$ which is $\sim 10 \%$ of FSR $=1.2 \mathrm{~nm}$. This could be critical for some applications. The possible ways to decrease polarization sensitivity are better fabrication conditions and an introduction of upper polymer cladding to compensate spectral shift with stress-induced birefringence. This approach was successfully implemented on SOI platform [23], however, polymers possess much lower mechanical stresses [2].

We also evaluated performance of proposed waveguides as a part of high speed transmission. Fig. 5a represents the experimental setup for a 28 Gbaud Nyquistshaped optical 64-QAM signal. The transmitter consists of two synchronized $50 \mathrm{GSa} / \mathrm{s}$ arbitrary waveform generator (AWG), optical IQ modulator and an external cavity laser (ECL) laser having less than $100 \mathrm{kHz}$ linewidth (LW). A pseudo-random bit sequence with a word length of $2^{15}-1$ (PRBS15) is generated and Gray mapped to generate a 28Gbaud 64QAM signal followed by Nyquist pulse shaping having a 0.15 roll off factor. The sequence is then resampled to match the sampling rate of the AWGs. At the output of IQ modulator optical signal is amplified using an erbium doped amplifier (EDFA). An optical signal to noise ratio (OSNR) adjusting module consisting of an optical attenuator and an automatic gain control EDFA with constant output power is used. Then signal is fed into preamplifier and band-pass filtered followed by the coherent receiver with an integrated local oscillator laser having less than $100 \mathrm{kHz}$ LW. A digital storage oscilloscope (DSO, $80 \mathrm{GSa} / \mathrm{s}, 33 \mathrm{GHz}$ ) is used to convert the signal into digital domain with offline demodulation. In Fig. 6 we present measured BER as a function of OSNR for optical back to back and transmission through $10 \mathrm{~cm}$ long spiral shape cylindrical waveguide (see Fig. 5b). In case of optical back to back, the waveguide is replaced with equivalent optical attenuator to have the same noise performance. As shown in Fig. 6, in both cases the 28 Gbaud 64-QAM signals achieve BER performance below the $20 \%$ overhead soft decision-forward error correction (20\% OH SD-FEC) threshold of $2 \times 10^{-2}$, yielding error-free post-FEC net bit rate of $140 \mathrm{Gbit} / \mathrm{s}$ per wavelength per polarization.

\section{CONCLUSIONS}

We experimentally demonstrate the method to notably decrease the propagation losses and the polarization sensitivity of polymer waveguides while maintaining a small device footprint. Our method also allows achieving sub $400 \mathrm{~nm}$ feature size with inexpensive contact lithography tool.
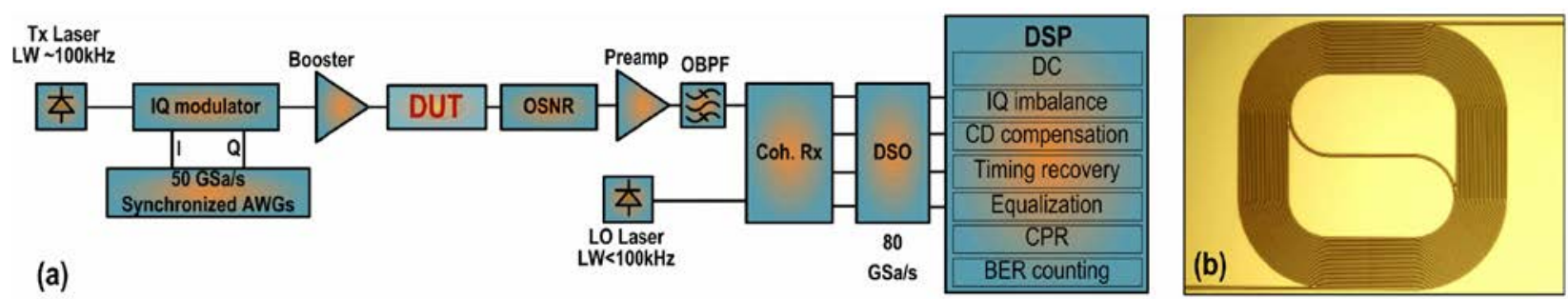

Fig. 5. 28Gbaud 64QAM transmission link scheme (a) and an optical microscopy image of the spiral-shape waveguide (b). 


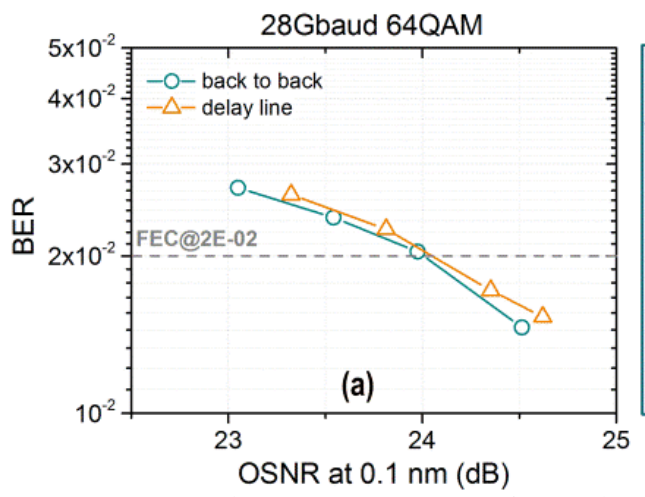

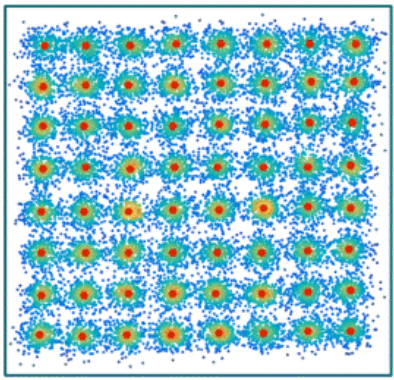

(b)

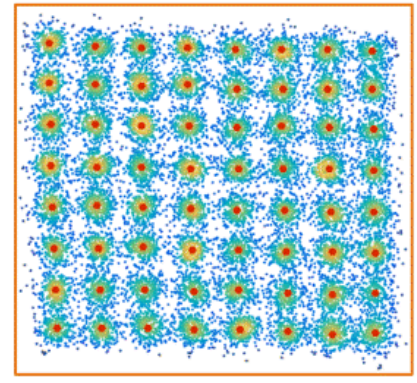

(c)

Fig. 6. BER vs OSNR (a) for 28 Gbaud 64-QAM signal for B2B (b) and the waveguide (c).

Enhanced performance of single waveguides and microring resonators is validated. Insignificant transmission penalty is observed for the high speed 28 Gbaud optical 64-QAM signals. Error-free 140 Gbit/s post-FEC net bit rate is demonstrated.

\section{REFERENCES}

[1] L. Eldada and L. W. Shacklette, "Advances in polymer integrated optics," IEEE J. Sel. Top. Quantum Electron., vol. 6, no. 1, pp. 54-68, 2000.

[2] H. Ma, A. K. Y. Jen, and L. R. Dalton, "Polymer-based optical waveguides: Materials, processing, and devices," Adv. Mater., vol. 14, no. 19, pp. 1339-1365, 2002.

[3] N. Bamiedakis et al., "40 Gb/s data transmission over a 1-mlong multimode polymer spiral waveguide for board-level optical interconnects," J. Lightwave Technol., vol. 33, no. 4, pp. 882-888, 2015.

[4] R. Dangel et al., "Polymer-waveguide-based board-level optical interconnect technology for datacom applications," IEEE Trans. Adv. Packag., vol. 31, no. 4, pp. 759-767, 2008.

[5] S. Y. Cheng, K. S. Chiang, and H. P. Chan, "Polarization dependence in polymer waveguide directional couplers," IEEE Photon. Technol. Lett., vol. 17, no. 7, pp. 1465-1467, 2005.

[6] D. De Felipe et al., "Recent Developments in PolymerBased Photonic Components for Disruptive Capacity Upgrade in Data Centers," J. Lightwave Technol., vol. PP, no. 99, 7571112, 2016.

[7] K. S. Chiang, "Development of optical polymer waveguide devices," in Proc. SPIE, 2010, vol. 7605.

[8] K. Yasuhara, S. Yoshida, F. Yu, and T. Ishigure, "Low-loss circular core single-mode polymer optical waveguide compatible with Si photonics chips for off-chip interconnects," in 5th IEEE Photonics Society Optical Interconnects Conference, San Diego, CA, 9-11 May 2016, pp. 88-89.

[9] T. Han, S. Madden, D. Bulla, and B. Luther-Davies, "Low loss chalcogenide glass waveguides by thermal nano-imprint lithography," Opt. Express, vol. 18, no. 18, pp. 1928619291, 2010

[10]C. Y. Chao and L. J. Guo, "Reduction of surface scattering loss in polymer microrings using thermal-reflow technique," IEEE Photon. Technol. Lett., vol. 16, no. 6, pp. 1498-1500, 2004.
[11]L. Chang et al., "Waveguide-coupled micro-ball lens array suitable for mass fabrication," Opt. Express, vol. 23, no. 17, pp. 22414-22423, 2015.

[12] S. C. Shen, C. T. Pan, H. P. Chou, Y. H. Chao, K. L. Lin, and J. Yang Jauh, "Batch assembly micro-ball lens array for Si-based optical coupling platform in free space," Proc. SPIE, vol. 4416, pp. 326-333, 2001.

[13]M. Hikita, R. Yoshimura, M. Usui, S. Tomaru, and S. Imamura, "Polymeric optical waveguides for optical interconnections," Thin Solid Films, vol. 331, no. 1-2, pp. 303-308, 1998

[14]A. J. Maker and A. M. Armani, "Low-loss silica-on-silicon waveguides," Opt. Lett., vol. 36, no. 19, pp. 3729-3731, 2011.

[15]X. Zhang and A. M. Armani, "Silica microtoroid resonator sensor with monolithically integrated waveguides," Opt. Express, vol. 21, no. 20, pp. 23592-23603, 2013.

[16]C. Y. Chao, W. Fung, and L. J. Guo, "Polymer microring resonators for biochemical sensing applications," IEEE J. Sel. Top. Quantum Electron., vol. 12, no. 1, pp. 134-142, 2006.

[17]A. Marinins, N. Knudde, and S. Popov, "Air-Suspended SU8 Strip Waveguides with High Refractive Index Contrast," IEEE Photon. Technol. Lett., vol. 28, no. 17, pp. 1862-1865, 2016.

[18]S. R. Lee, M. R. Kim, E. H. Jo, and K. B. Yoon, "Synthesis of very low birefringence polymers using fluorinated macromers for polymeric waveguides," High Perform. Polym., vol. 28, no. 2, pp. 131-139, 2016.

[19]J. Scheuer and A. Yariv, "Fabrication and characterization of low-loss polymeric waveguides and micro-resonators," $J$. Eur. Opt. Soc., vol. 1, 2006.

[20]J. K. S. Poon, L. Zhu, G. A. DeRose, and A. Yariv, "Polymer microring coupled-resonator optical waveguides," J. Lightwave Technol., vol. 24, no. 4, pp. 1843-1849, 2006.

[21]R. Morarescu et al., "Fabrication and Characterization of High-Optical-Quality-Factor Hybrid Polymer Microring Resonators Operating at Very Near Infrared Wavelengths," IEEE Photon. J., vol. 8, no. 2, 2016.

[22]D. Dai, B. Yang, L. Yang, Z. Sheng, and S. He, "Compact microracetrack resonator devices based on small SU-8 polymer strip waveguides," IEEE Photon. Technol. Lett., vol. 21, no. 4, pp. 254-256, 2009.

[23]D. X. Xu, S. Janz, and P. Cheben, "Design of polarizationinsensitive ring resonators in silicon-on-insulator using MMI couplers and cladding stress engineering," IEEE Photon. Technol. Lett., vol. 18, no. 2, pp. 343-345, 2006. 